\title{
Rhopalocnemis phalloides has one of the most reduced and mutated plastid genomes known
}

\author{
Mikhail I Schelkunov ${ }^{\text {Corresp., 1, } 2 \text {, Maxim S Nuraliev }}{ }^{3,4}$, Maria D Logacheva ${ }^{1,5}$ \\ ${ }^{1}$ Skolkovo Institute of Science and Technology, Moscow, Russia \\ 2 Institute for Information Transmission Problems, Moscow, Russia \\ 3 Faculty of Biology, Moscow State University, Moscow, Russia \\ 4 Joint Russian-Vietnamese Tropical Scientific and Technological Center, Cau Giay, Hanoi, Vietnam \\ ${ }^{5}$ A.N. Belozersky Research Institute of Physico-Chemical Biology, Moscow State University, Moscow, Russia \\ Corresponding Author: Mikhail I Schelkunov \\ Email address: shelkmike@gmail.com
}

Although most plant species are photosynthetic, several hundred species have lost the ability to photosynthesize and instead obtain nutrients via various types of heterotrophic feeding. Their plastid genomes markedly differ from the plastid genomes of photosynthetic plants. In this work, we describe the sequenced plastid genome of the heterotrophic plant Rhopalocnemis phalloides, which belongs to the family Balanophoraceae and feeds by parasitising other plants. The genome is highly reduced (18,622 base pairs versus approximately 150 kilobase pairs in autotrophic plants) and possesses an extraordinarily high AT content, 86.8\%, which is inferior only to AT contents of plastid genomes of Balanophora, a genus from the same family. The gene content of this genome is quite typical of heterotrophic plants, with all of the genes related to photosynthesis having been lost. The remaining genes are notably distorted by a high mutation rate and the aforementioned AT content. The high AT content has led to sequence convergence between some of the remaining genes and their homologues from AT-rich plastid genomes of protists. Overall, the plastid genome of $R$. phalloides is one of the most unusual plastid genomes known. 


\section{Rhopalocnemis phalloides has one of the most reduced} 2 and mutated plastid genomes known

4 Mikhail I. Schelkunov ${ }^{1,2}$, Maxim S. Nuraliev ${ }^{3,4}$, Maria D. Logacheva $^{1,5}$

$5{ }^{1}$ Skolkovo Institute of Science and Technology, Moscow, Russia

$6 \quad 2$ Institute for Information Transmission Problems, Moscow, Russia

$7{ }^{3}$ Faculty of Biology, Moscow State University, Moscow, Russia

$8{ }^{4}$ Joint Russian-Vietnamese Tropical Scientific and Technological Center, Cau Giay, Hanoi, 9 Vietnam

105 A.N. Belozersky Research Institute of Physico-Chemical Biology, Moscow State University, 11 Moscow, Russia

13 Corresponding Author:

14 Mikhail Schelkunov

15 Email address: shelkmike@gmail.com 
16

17

18

19

20

21

22

23

24

25

26

27

28

29

30

31

32

33

34

35

36

37

38

39

40

41

42

43

44

45

46

47

48

49

50

51

52

53

54

55

56

\section{Abstract}

Although most plant species are photosynthetic, several hundred species have lost the ability to photosynthesize and instead obtain nutrients via various types of heterotrophic feeding. Their plastid genomes markedly differ from the plastid genomes of photosynthetic plants. In this work, we describe the sequenced plastid genome of the heterotrophic plant Rhopalocnemis phalloides, which belongs to the family Balanophoraceae and feeds by parasitising other plants. The genome is highly reduced (18,622 base pairs versus approximately 150 kilobase pairs in autotrophic plants) and possesses an extraordinarily high AT content, $86.8 \%$, which is inferior only to AT contents of plastid genomes of Balanophora, a genus from the same family. The gene content of this genome is quite typical of heterotrophic plants, with all of the genes related to photosynthesis having been lost. The remaining genes are notably distorted by a high mutation rate and the aforementioned AT content. The high AT content has led to sequence convergence between some of the remaining genes and their homologues from AT-rich plastid genomes of protists. Overall, the plastid genome of $R$. phalloides is one of the most unusual plastid genomes known.

\section{Introduction}

Though plants are generally considered photosynthetic organisms, there are several hundred plant species that have lost the ability to photosynthesize during the course of evolution (Westwood et al., 2010; Merckx et al., 2013). They feed either by parasitising other plants or by obtaining nutrients from fungi.. In addition to the completely heterotrophic plants, there are also plants that combine the ability to photosynthesize with the heterotrophic lifestyle. They are termed partial heterotrophs (or hemi-heterotrophs, or mixotrophs) in contrast to the former, which are termed complete heterotrophs (or holo-heterotrophs).

The completely heterotrophic plants show a high degree of similarity, though there were several dozen cases of independent transition to complete heterotrophy. For example, these plants all either lack leaves or have very reduced leaves. These plants are non-green because of the absence (or at least highly reduced amounts (Cummings \& Welschmeyer, 1998)) of chlorophyll. Additionally, a common feature of many completely heterotrophic angiosperms is that they spend most of their lifetimes underground, since without the need to photosynthesize their only reason to appear aboveground is for flowering and seed dispersal.

Genomic studies of heterotrophic plants are mostly focused on plastid genomes, since 1) most of the plastid genes are related to photosynthesis, and thus changes in the plastid genomes are expected to be more prominent compared to mitochondrial and nuclear genomes, and 2) plastid genomes are smaller than nuclear and mitochondrial ones and usually have higher copy numbers and are thus easier to sequence (Daniell et al., 2016; Gualberto \& Newton, 2017; Sakamoto \& Takami, 2018). The main feature of the plastid genomes of complete heterotrophs is the loss of genes responsible for photosynthesis and respective shortening of the genomes, from approximately $150 \mathrm{kbp}$ (typical of autotrophic plants) to, in the most extreme known case, 12 kbp (Bellot \& Renner, 2015; Graham, Lam \& Merckx, 2017; Wicke \& Naumann, 2018). The remaining genes are the ones with functions not related to photosynthesis. Usually they are $a c c D$ 
57 (a gene whose product participates in fatty acid synthesis - one of the plastid functions besides 58 photosynthesis), $\operatorname{clp} P$ (encodes a component of a complex responsible for degradation of waste proteins in plastids), $y c f 1$ (thought to encode a component of the translocon - the complex which imports proteins from cytoplasm into plastids), $y c f 2$ (a conserved gene present in almost all plants, but with unknown function) and various genes required for translation of the aforementioned ones, namely genes that code for protein and RNA components of the plastid ribosome and for tRNAs. One of the tRNA-coding genes, trnE-UUC, also has an additional function, with its product participating in haem synthesis (Kumar et al., 1996).

In addition to the expected shortening of the genome, there are some peculiar and still unexplained features in the plastid genomes of heterotrophic plants, namely their increased mutation accumulation rate (Bromham, Cowman \& Lanfear, 2013; Wicke \& Naumann, 2018) and increased AT content (Wicke \& Naumann, 2018). In the most extreme cases, plastid genomes of heterotrophic plants may accumulate mutations approximately 100 times faster than their closest autotrophic relatives (Bellot \& Renner, 2015). The most obvious explanation, the relaxation of selection, is refuted by the fact that $\mathrm{dN} / \mathrm{dS}$ (a common measure of selective pressure) is usually not increased in the plastid genes of heterotrophic plants, except for photosynthesis-related genes during their pseudogenization, but the mutation accumulation rate is high even after the loss of all such genes (Logacheva, Schelkunov \& Penin, 2011; Barrett et al., 2014; Schelkunov et al., 2015; Lam, Soto Gomez \& Graham, 2015; Wicke et al., 2016; Naumann et al., 2016). AT content is increased from approximately $65 \%$ in autotrophic species (Smith, 2012) to $88.4 \%$ in the most prominent case among heterotrophic species (Su et al., 2019), also because of an unknown reason.

Genes not related to photosynthesis, such as $a c c D$ and $\inf A$, are sometimes transferred to the nuclear genome (Millen et al., 2001; Rousseau-Gueutin et al., 2013; Liu et al., 2016). Therefore, when all genes with functions not related to translation are transferred to the nuclear genome, there will be no reasons to keep the translation apparatus in plastids, and the genes responsible for translation will also be lost. Thus, the plastid genome is potentially able to disappear entirely. Indeed, two putative cases of the complete plastid genome loss are known: one in algae of the genus Polytomella (Smith \& Lee, 2014) and the other one in the parasitic plant Rafflesia lagascae (Molina et al., 2014); the second case is disputable (Krause, 2015).

The initial aim of the present study was to prove that the completely heterotrophic plant Rhopalocnemis phalloides had also lost its plastid genome completely. Rhopalocnemis phalloides is a parasitic plant from the family Balanophoraceae (order Santalales) which occurs in Asia and feeds by obtaining nutrients from roots of various plants. Initially we sequenced approximately 10 million pairs of reads on the HiSeq 2000 platform and observed no contigs with similarity to typical plastid genes, while there were obvious mitochondrial contigs. Based on our experience in studying plastid genomes of heterotrophic plants, mitochondrial contigs usually have lower sequencing coverage than plastid contigs; thus, the plastid genome is always easier to assemble. This led us to suppose that the plastid genome in $R$. phalloides may have been completely lost. To verify this, we sequenced approximately 200 million pairs of additional reads. What we found is that the plastid genome is in fact present, but its tremendous AT content 
98 (86.8\%) hampered PCR, which is one of the usual steps in library preparation of Illumina, and

99 thus the sequencing coverage of the genome was much lower than one might have expected. This

100 article is dedicated to the analysis of this plastid genome.

101

102

\section{Materials \& Methods}

103

104

Sample collection and sequencing

105

106

The specimen of $R$. phalloides was collected during an expedition of the Russian-Vietnamese

107 Tropical Centre in Kon Tum province, Vietnam, in May 2015 (voucher information: Southern Vietnam, Kon Tum prov., Kon Plong distr., Thach Nham protected forest, $17 \mathrm{~km} \mathrm{~N}$ of Mang Den

109 town, in open forest, N 14 $45^{\prime} 15^{\prime \prime}$ E $108^{\circ} 17^{\prime} 40^{\prime \prime}$, elev. 1400 m, Nuraliev M.S., Kuznetsov A.N., Kuznetsova S.P., No. 1387, 18.04.2015). The studied material was preserved in silica gel and in RNAlater. The voucher is deposited at the Moscow University Herbarium (MW) (Seregin, 2018) with the barcode MW0755444.

113 DNA was extracted from an inflorescence using a CTAB-based method (Doyle, 1987), and the

114 DNA library was prepared using the NEBNext DNA Ultra II kit (New England Biolabs).

115 Sequencing was performed with a NextSeq 500 sequencing machine (Illumina) in the paired end mode, producing 387,351,294 reads (193,675,647 read pairs), each 150 bp long.

RNA was extracted from an inflorescence using the RNeasy Mini kit (Qiagen). Plastid transcripts are usually not polyadenylated, so the method of poly(A) RNA selection was not applicable in our study. Instead, we used a protocol based on depletion of ribosomal RNA with the Plant Leaf Ribo Zero kit (Illumina). The RNA-seq library was prepared using the NEBNext RNA Ultra II kit (New England Biolabs) and sequenced on a HiSeq 2500 sequencing machine (Illumina) with TruSeq reagents v.4 in the paired end mode, producing 54,794,466 reads $(27,397,233$ read pairs), 125 bp each.

\section{Genome assembly and annotation}

Both DNA-seq and RNA-seq reads were trimmed by Trimmomatic 0.32 (Bolger, Lohse \& Usadel, 2014) in the palindromic mode, removing bases with quality less than 3 from the 3 ' ends of reads, and fragments starting from 4-base-long windows with average quality less than 15 (SLIDINGWINDOW:4:15). Reads that, after trimming, had average quality less than 20 or length shorter than 30 bases were removed.

132 The assembly was performed from DNA-seq reads by two tools. First, it was made by CLC

133 Assembly Cell 4.2 (https://www.qiagenbioinformatics.com/products/clc-assembly-cell/) with the 134 default parameters. Second, it was made by Spades 3.9.0 (Bankevich et al., 2012). Because the 
135 performance of Spades is slow when running on large number of reads, prior to starting its 136 assembly we removed from reads k-mers with coverage less than $50 \times$ by Kmernator 1.2.0 137 (https://github.com/JGIBioinformatics/Kmernator). This allowed us to eliminate most reads 138 belonging to the nuclear genome (and, potentially, some reads belonging to low-covered plastid 139 regions), thus highly reducing the number of reads. The Spades assembly was run on this 140 reduced read set, with the "--only-assembler" and "--careful" options. To determine the read 141 coverage of contigs in these two assemblies, we aligned to them reads by CLC Assembly Cell

1424.2 , requiring at least $80 \%$ of the length of each read to align with a sequence similarity of at 143 least $98 \%$.

144 To find contigs potentially belonging to plastid and mitochondrial genomes, we aligned by 145 BLASTN and TBLASTN from BLAST 2.3.0+ suit (Camacho et al., 2009) proteins and non146 coding RNA (ncRNA) genes from reference species. As the references, we used sequences from 147 the plastid genomes of Balanophora reflexa (NCBI accession KX784266), Balanophora 148 laxiflora (NCBI accession KX784265), Viscum album (NCBI accession NC_028012), Osyris 149 alba (NCBI accession NC_027960), Arabidopsis thaliana (NCBI accession NC_000932),

150 Nicotiana tabacum (NCBI accession NC_018041) and mitochondrial genomes of Viscum album 151 (NCBI accession NC_029039), Citrullus lanatus (NCBI accession GQ856147), Mimulus 152 guttatus (NCBI accession NC_018041). Balanophora, Viscum album and O. alba were used 153 because they, like $R$. phalloides, belong to Santalales. Other species were chosen because they 154 belong to other orders of eudicots. Alignment was performed with the maximum e-value of $10^{-3}$ 155 and low complexity filter switched off. The word size was 7 for BLASTN and 3 for TBLASTN. 156 Here and later, the local BLAST was used with the parameter "max_target_seqs" set to $10^{9}$ to 157 avoid the problem discussed by Shah et al. (2018), who state that BLAST results may be 158 improper when this parameter is set to a small value.

159 Five contigs containing plastid genes were found in the CLC assembly and three contigs in the 160 Spades assembly. After aligning contigs of these two assemblies to each other (BLASTN, 161 maximum e-value $10^{-3}$, word size 7 , low complexity filter switched off), it appeared that the 162 places at which the CLC contigs were broken by gaps corresponded to continuous places in the 163 Spades contigs and, vice versa, gaps in the Spades contigs corresponded to continuous places in 164 the CLC contigs. This allowed us, by joining the contigs of these two assemblies, to create a 165 circular sequence corresponding to the plastid genome. To check the assembly, we mapped reads 166 (in CLC Assembly Cell 4.2, requiring at least 80\% of the length of each read to align with a 167 sequence similarity of at least 98\%) to the resultant sequence and verified (by eye, in CLC 168 Genomics Workbench 7.5.1, https://www.qiagenbioinformatics.com/products/clc-genomics169 workbench/) that there were no places uncovered by reads and no places where the insert size 170 abruptly decreased or increased. Such places of abrupt increase or decrease of the insert size may 171 indicate regions with assembly errors, consisting of sequence insertions or deletions, 172 respectively. As read mapping is complicated on the edges of a sequence, we also performed 173 such analysis on a reoriented version of the plastid genome, in which the sequence was broken in 174 the middle and the ends were joined. These analyses indicated that the assembly contained no 175 errors. 
176 To find genes in the plastid genome, we used a complex strategy, because highly mutated genes

177 may be hard to notice. We used the following methods:

178 1. The alignment of reference protein-coding and ncRNA-coding genes by BLASTN and

179 TBLASTN, as described above.

180

181

182

183

184

185

186

187

188

189

190

191

192

193

194

195

196

197

198

199

200

201

202

203

204

205

206

207

208

209

210

211

212

213

214

215

216

2. Open reading frames were scanned by InterProScan 5.11 (Jones et al., 2014) using the InterPro 51.0 (Finn et al., 2017) database with the default parameters. "Open reading frames" here were any sequences at least 20 codons long uninterrupted by stop codons. Not requiring an ORF to begin from a start-codon allowed for the detection of exons in multi-exonic genes.

3. The genome was scanned by Infernal 1.1.2 (Nawrocki \& Eddy, 2013) with RNA models from Rfam 12.2 database (Nawrocki et al., 2015) to predict ncRNA-coding genes. The maximum allowed e-value was set to $10^{-3}$.

4. To predict rRNA-coding genes, RNAmmer 1.2 server (Lagesen et al., 2007) was used in bacterial mode and eukaryotic mode.

5. The genome was scanned by tRNAscan-SE 1.23 (Lowe \& Eddy, 1997) with the default parameters, in the organellar (models trained on plastid and mitochondrial tRNAs) and also in the general (models trained on tRNAs from all three genomes) mode, to predict tRNA-coding genes.

6. The genome was annotated by DOGMA (Wyman, Jansen \& Boore, 2004) and Verdant (McKain et al., 2017).

7. When determining which ATG codon was a true start codon, we compared the sequence of a gene with sequences of its homologs from the aforementioned reference species.

8. To determine exon borders, RNA-seq reads with a minimum length of $100 \mathrm{bp}$ (to minimize false mappings) were mapped to the genome by CLC Assembly Cell 4.2, requiring at least $50 \%$ of each read's length to map with a sequence similarity of at least $90 \%$. Exon borders were found by eye in CLC Genomics Workbench 7.5.1 as regions of genes in which there were many partially mapped reads. The exon borders of the reference species were used for comparison.

9. To check for RNA editing that could create new start or stop codons, we mapped RNAseq reads with a minimum length of 100 bp by CLC Assembly Cell 4.2, requiring at least $80 \%$ of each read's length to map with a sequence similarity of at least $90 \%$. Mismatches between the reads and the genome were inspected by eye in CLC Genomics Workbench 7.5.1.

10. After annotating the genes, we additionally verified that there were no remaining regions with high sequence complexity, relatively low AT content or high coverage by RNA-seq reads where no genes were predicted. Regions with high sequence complexity were predicted in the genome by CLC Genomics Workbench 7.5.1 using K2 algorithm (Wootton \& Federhen, 1993) with a window size of $101 \mathrm{bp}$. The AT content plot was created by a custom script with 200-bp-long windows. RNA-seq reads with a minimum length of $100 \mathrm{bp}$ were mapped by CLC Assembly Cell 4.2, requiring at least $80 \%$ of each read's length to map with a sequence similarity of at least $90 \%$. 
217 After completing gene prediction, the plastid genome was reoriented to start from the first

218 position of rps 14, as this is the first gene in the canonical representation of the plastid genome of

219 A. thaliana which is also present in the plastid genome of $R$. phalloides.

220

221

222

223

224

225

226

227

228

229

230

231

232

233

234

235

236

237

238

239

240

241

242

243

244

245

246

247

248

249

250

251

252

253

254

255

\section{Estimation of contamination amount}

The nuclear genome size could be overestimated if, in addition to the own DNA of $R$. phalloides, contaminating DNA was sequenced. For example, this contamination may originate from endophytic bacteria and fungi. To estimate the amount of contamination, 1000 random DNA-seq read pairs, taken after the trimming, were aligned by BLAST to NCBI databases. Taxonomies of their best matches were used as proxies for the reads' source taxonomies. To increase the sensitivity of the search, the analysis was performed as follows:

1. All reads were aligned to NCBI NT (the database current as of September 18, 2017) by BLASTN from BLAST 2.3.0+ suite with the maximum allowed e-value of $10^{-3}$ and the word size of $7 \mathrm{bp}$. To decrease the number of false-positive matches, hard masking of low-complexity regions ("-soft_masking false" option) was used.

2. All reads were aligned to NCBI NR (the database current as of September 18, 2017) by BLASTX from BLAST 2.3.0+ suite with the maximum allowed e-value of $10^{-3}$ and the word size of $3 \mathrm{bp}$. Hard masking in BLASTX is enabled by default.

3. If at least one of two reads in a pair had matches to NT, the taxonomy of the match with the lowest e-value was considered the taxonomy of the read pair. If the read pair had no matches in NT, the taxonomy of the match to NR with the lowest e-value was considered the taxonomy of the read. Therefore, the alignment to NT had higher priority than the alignment to NR. This was done to take into account synonymous positions of genes, where possible, and thus increase the precision of the taxonomic assignment of read pairs.

\section{Other analyses}

To determine the phylogenetic placement of $R$. phalloides within Balanophoraceae, we utilised the alignment of genes from 186 species (180 species of Santalales plus 6 outgroup species) created by Su et al. (2015). Rhopalocnemis phalloides was not studied in that article. Seven genes were used for the phylogenetic analysis in that work: plastid $a c c D$, matK, $r b c L$; nuclear $18 \mathrm{~S}$ rDNA, 26S (also known as 25S) rDNA and RPB2; and mitochondrial matR. As matK and $r b c L$ are absent from the plastid genome of $R$. phalloides, we were unable to use them. accD of $R$. phalloides contains many mutations and thus can be aligned improperly, so we did not use it either. Mitochondrial mat $R$ is disrupted in $R$. phalloides by several frameshifting indels. Owing to the large size of the nuclear genome of $R$. phalloides (see the paragraph "Other genomes of $R$. phalloides"), RPB2 had a low coverage, and its sequence could not be obtained from the 
256 available DNA-seq reads. The sequences of $18 \mathrm{~S}$ rDNA and $26 \mathrm{~S}$ rDNA were easier to determine, 257 as they had many copies in the nuclear genome and thus their coverage was higher. To find their 258 sequences among the contigs, we aligned $18 \mathrm{~S}$ rDNA and $26 \mathrm{~S}$ rDNA of $A$. thaliana by BLASTN

259

260

261

262

263

264

265

266

267

268

269

270

271

272

273

274

275

276

277

278

279

280

281

282

283

284

285

286

287

288

289

290

291

292

293

294

295

296 with the default parameters to the contigs of the Spades assembly. The sequences of 18S rDNA and 26S rDNA were added to the alignment of Su et al. (2015) using MAFFT 7.402 (Katoh \& Standley, 2013) with options --addfragments and --maxiterate 1000. The phylogenetic tree was built with RAxML 8.2.4 (Stamatakis, 2014), utilising 20 starting stepwise-addition parsimony trees, employing GTR+Gamma model, with the same 6 outgroup species as in the work of Su et al. (2015) (Antirrhinum majus, A. thaliana, Camellia japonica, Cornus florida, Myrtus communis and Spinacia oleracea). The required number of bootstrap pseudoreplicates was determined by RAxML automatically with the extended majority-rule consensus tree criterion ("autoMRE"). The tree was visualised with FigTree 1.4.3 (http://tree.bio.ed.ac.uk/software/figtree/).

To compare the substitution rate in the plastid genome of $R$. phalloides with substitution rates in other species of Santalales, we used common protein-coding genes of $R$. phalloides, B. reflexa, A. thaliana (used as the outgroup) and the only four species of Santalales with published plastid genomes as of 2017: V. album, O. alba, Champereia manillana and Schoepfia jasminodora. Their protein-coding gene alignment was created by TranslatorX 1.1 (Abascal, Zardoya \& Telford, 2010) based on an alignment of the corresponding amino acid sequences performed by Muscle 3.8.31 (Edgar, 2004) with the default parameters. $y c f 1, y c f 2$ and $a c c D$ of R. phalloides differed from the homologous genes of other species so much that a reliable alignment was not possible. Alignments of other genes were then concatenated into a single alignment and provided to Gblocks server (Castresana, 2000), which removed poorly aligned regions from the alignment. Gblocks was run in the codon mode, with the default parameters. Substitution rates and selective pressure were evaluated by codeml from PAML 4.7 (Yang, 2007) with the F3×4 codon model, starting $\mathrm{dN} / \mathrm{dS}$ value of 0.5 and starting transition/transversion rate of 2 . The phylogenetic tree provided to PAML was a subtree of the large phylogenetic tree of Santalales, produced as described above. Additionally, the analysis of substitution rates and selective pressure was performed by BppSuite 2.3.2 (Guéguen et al., 2013). To the best of our knowledge, this is the only tool that is capable of phylogenetic analyses of protein-coding sequences that takes into account different codon frequencies in different sequences (Guéguen \& Duret, 2017), whereas PAML uses a single averaged codon frequency for all sequences. This is important, because the codon frequencies in $R$. phalloides and B. reflexa highly differ from the codon frequencies in the mixotrophic Santalales of comparison. The program bppml from BppSuite was run using a nonhomogeneous ("one_per_branch") model, the substitution model was YN98, the codon model $\mathrm{F} 3 \times 4$, starting $\mathrm{dN} / \mathrm{dS}$ values of 0.5 and starting transition/transversion rates of 2 . Starting branch lengths were 0.1 substitutions per codon. The parameter estimation was performed by the full-derivatives method with optimization by the Newton-Raphson method ("optimization=FullD(derivatives=Newton)"), using parameters transformation ("optimization.reparametrization=yes").

To check for similarity between the genes and the proteins of the $R$. phalloides plastid genome and sequences from other species, we performed BLASTN and BLASTP alignment against 
297 NCBI NT and NR databases, respectively, on the NCBI website

298 (https://blast.ncbi.nlm.nih.gov/Blast.cgi) on March 4, 2018 with the default parameters.

299 To build the phylogenetic tree of $r r n 16$, we took sequences of $r r n 16$ from plastid genomes of

300 Embryophyta and SAR ("Stramenopiles, Alveolata, Rhizaria", a clade of protists), one random

301 representative per order. The plastid genomes were taken from the RefSeq database (current as

302 of May 12, 2019), the taxonomy from NCBI Taxonomy (current as of May 13, 2019). Overall,

303 there were 93 representatives of Embryophyta and 35 representatives of SAR. To these

304 sequences we added the sequences of rrn16 from $R$. phalloides, Corynaea crassa (NCBI

305 accession U67744), Balanophora japonica (NCBI accession KC588390), Nitzschia sp. IriIs04

306 (NCBI accession AB899709) and Plasmodium cynomolgi (NCBI accession AB471804). These

307 particular sequences of Nitzschia and Plasmodium were randomly chosen among the SAR

308 sequences that produced matches to the rrn 16 of $R$. phalloides in the BLAST search described in

309 the previous paragraph. There were also matches to Leucocytozoon in that analysis, but

310 Leucocytozoon caulleryi had already been randomly chosen as a representative for the SAR

311 order Haemosporida, so there was no need to add it again. The sequences of these 133 rrn16

312 genes were aligned by MAFFT 7.402 with the option --maxiterate 1000. Poorly aligned regions

313 were removed by Gblocks server with the option "Allow gap positions within the final blocks"

314 switched on. Without this option Gblocks is too severe for alignments which contain many

315 sequences. The unrooted phylogenetic tree was built by RAxML 8.2.4 with the parameters

316 described above. Branches corresponding to clades that had bootstrap support values below 70

317 were collapsed by TreeGraph 2.14.0-771 beta (Stöver \& Müller, 2010). The tree was visualised

318 by FigTree 1.4.3.

319 Codon usage and amino acid usage of the common protein-coding genes of $R$. phalloides and

320 species of comparison were calculated by CodonW 1.4.2 (Peden, 1999). Frequencies of 21-bp-

321 long k-mers were calculated for the trimmed DNA-seq reads by Jellyfish 2.1.2 (Marçais \&

322 Kingsford, 2011), not using the Bloom filter (to count the number of low-frequency k-mers

323 precisely).

324 The list of plastid genomes with their lengths and AT contents was obtained from the NCBI

325 database (https://www.ncbi.nlm.nih.gov/genome/browse/\#!/organelles/). Information on whether

326 a specific plant species is completely heterotrophic was obtained by literature analysis.

327

328

Results \& Discussion

329

The gene content of the $R$. phalloides plastid genome

331

332 The plastid genome of $R$. phalloides is circular-mapping and has a length of $18,622 \mathrm{bp}$ long. Its

333 map is represented in Fig. 1, in a linear form, for convenience. The protein-coding gene content

334 is quite typical for highly reduced plastid genomes of completely heterotrophic plants (Graham,

335 Lam \& Merckx, 2017; Wicke \& Naumann, 2018). The plastid genome of $R$. phalloides possesses

PeerJ reviewing PDF | (2018:12:33547:2:0:ACCEPTED 8 Jul 2019) 
336 the genes $a c c D, c l p P, y c f 1, y c f 2$ (mentioned in the Introduction) and 9 genes encoding protein 337 components of the ribosome. Additionally, it codes for $r r n 16$ and $r r n 23$, RNA components of the 338 plastid ribosome. Like several other highly reduced plastid genomes, it lacks $r r n 4.5$ and $r r n 5-$ genes coding for two other RNAs of the ribosome - which poses the interesting puzzle of how the ribosome works without these genes in the plastid genome. One possibility is that these genes were transferred either to the mitochondrial or to the nuclear genome and are now transcribed there and imported to the plastids from the cytoplasm. The other possibility is that the ribosome is capable of working without them, akin to how it can work without some ribosomal proteins (Tiller \& Bock, 2014). We plan to clarify this question in an upcoming article dedicated to the analysis of the $R$. phalloides transcriptome.

The tRNA-coding gene content of the $R$. phalloides plastid genome is also puzzling. The standard method to predict tRNA-coding genes is the program tRNAscan-SE. It has a dedicated "organellar" mode in which tRNA models were trained on mitochondrial- and plastid-encoded tRNA sequences and structures. It also has a "general" mode whose models are based on nuclearencoded tRNAs. In the organellar mode, the tool predicts 64 tRNA-coding genes, which is much more than the approximately 30 tRNA-coding genes encoded in plastomes of typical autotrophic species (Wicke et al., 2011). In the general mode, the tool predicts zero tRNA-coding genes. Our experience in working with different plastid genomes suggests that results of predictions in these two modes usually coincide. Of the 64 predicted tRNA-coding genes, 61 have introns, and the mean AT content of the 64 genes is $94 \%$. Therefore, we supposed that most of them, if not all, were false-positive predictions. They could originate from the ease with which sequences of low complexity form secondary structures - these spuriously generated cloverleaf-like structures may have deceived the algorithms of tRNAscan-SE. Seventeen of the predicted tRNA-coding genes were for isoleucine tRNAs, and 11 were for lysine. This further attested to the false-positive nature of these genes, as false-positively predicted tRNA-coding genes in an AT-rich genome are expected to have AT-rich anticodons, and the anticodons of isoleucine and lysine tRNAs are two of the most AT-rich of all amino acid anticodons. Of the three tRNA-coding genes without introns, one has an AT content of $76 \%$, another $92 \%$ and the third $96 \%$. Because of the relatively low AT, the first seems to be a possible candidate for a true gene. Its AT content was not only the lowest among the three predictions that do not have introns but also among all 64 predicted tRNA-coding genes. This is a $t r n L$ gene with anticodon TAA (UAA). Nevertheless, we could not confidently determine whether this gene was a false-positive prediction so we did not use it for any analyses. The only predicted $\operatorname{trn} E$ gene had an AT content of $99 \%$ and is thus very likely to be a false prediction. Therefore, the plastid genome of $R$. phalloides probably lost its trnE, like the plastid genomes of completely heterotrophic plants from the genus Pilostyles (Bellot \& Renner, 2015), although earlier trnE was deemed indispensable because of its function in haem synthesis (Howe \& Smith, 1991; Barbrook, Howe \& Purton, 2006). In the plastid genomes of Balanophora, the other genus of Balanophoraceae for which completely sequenced plastid genomes are available, $\operatorname{trnE}$ is present but is supposed to participate in haem synthesis only, having lost its function in translation (Su et al., 2019). The predicted trnE of $R$. phalloides is located in the intergenic region between ycfl and $r r n 23$, not where it is in Balanophora, which is an additional argument for the false-positive nature of this prediction. Potentially, in $R$. 
378

379

380

381

382

383

384

385

386

387

388

389

390

391

392

393

394

395

396

397

398

399

400

401

402

403

404

405

406

407

408

409

410

411

412

413

414

415

416

phalloides, trnE could have been transferred to the nuclear or the mitochondrial genome, transcribed there and imported into the plastids from the cytoplasm.

Overall, the gene content of the plastid genome of $R$. phalloides is similar to the gene content of the plastid genomes of Balanophora. All differences between them lie in differential losses of genes participating in translation, except for the loss of trnE in Rhopalocnemis, that function also in haem synthesis. Compared to Balanophora, R. phalloides lacks rps2, rps4, rps11, rpl14, trnE, rrn4.5, while Balanophora lacks rpll6 and rpl36 which are present in $R$. phalloides.

Most plastid genes of $R$. phalloides are shorter than their homologues in close mixotrophic relatives, although not as short as homologues in Balanophora (Table S1). The compaction of non-coding regions in the plastid genome in $R$. phalloides is also not as pronounced as in Balanophora, with $78.5 \%$ being coding (i.e. non-intergenic and non-intronic) in the plastid genome of R. phalloides and $94.2 \%$ and $95.2 \%$ in the plastid genomes of B. reflexa and $B$. laxiflora, respectively. Several genes of $R$. phalloides overlap. Namely, $y c f 1$ and $y c f 2$ overlap; so does $r p l 36$ which overlaps with both $r p s 7$ and $r p l 16$ (Fig. 1). The intron loss in R. phalloides is also not as pronounced as in Balanophora, with four cis-spliced and one trans-spliced introns remaining in $R$. phalloides, whereas only the trans-spliced intron remains in Balanophora, with all cis-spliced introns lost.

The gene order in the plastid genome of $R$. phalloides is neither collinear with the gene order of Balanophora, nor it is collinear with the typical gene order of photosynthetic plants (Table S2). Namely, the plastid genome of $R$. phalloides has 7 collinear blocks with the plastid genomes of Balanophora and 4 collinear blocks with the plastid genome of $A$. thaliana.

\section{The plastid genome of $R$. phalloides has a very high AT content}

One of the most interesting features of the $R$. phalloides plastid genome is its AT content of $86.8 \%$. Among plant genomes, it is surpassed only by the plastid genomes of $B$. reflexa and $B$. laxiflora, two close relatives of $R$. phalloides, which have an AT content of $88.4 \%$ and $87.8 \%$, respectively (Su et al., 2019). Among prokaryotes and eukaryotes other than plants, there are also several other known genomes with higher AT content, all belonging to mitochondria or apicoplasts, with the record held by the mitochondrial genome of a fungus, Nakaseomyces bacillisporus CBS 7720 (Bouchier et al., 2009), with the AT content of $89.1 \%$ (according to the NCBI site, information current as of June 14, 2018).

The increased AT content is a common feature of plastid genomes of completely heterotrophic plants (Fig. 2, Table S3); to the best of our knowledge, it remains unexplained. It correlates with the degree of plastid genome reduction, with plants whose plastid genomes are the most AT rich having simultaneously some of the smallest plastid genomes.

It was the high AT content which prevented us from detecting the plastid genome of $R$. phalloides from the initial assembly made of approximately 10 million read pairs. High AT content hampers PCR (Benjamini \& Speed, 2012), and as library preparation for Illumina 
417 sequencing machines usually involves PCR, coverage of AT-rich regions is decreased. When we 418 assembled the genome using an insufficient number of reads, the genome's sequence was broken 419 into multiple contigs containing regions with relatively low AT content. The breaks occurred in

420

421

422

423

424

425

426

427

428

429

430

431

432

433

434

435

436

437

438

439

440

441

442

443

444

445

446

447

448

449

450

451

452

453

454

455

456

457 the regions in which the AT content was higher; therefore, the coverage in those regions was decreased the most. The obtained sequences were not enough to determine whether the plastid genome was present because (as we describe further below) the sequences were usually similar to those from taxons other than plants, owing to the high AT content and high mutation accumulation rate. Therefore, we initially thought that these short contigs were horizontal transfers located in the mitochondrial genome. Increasing the number of reads allowed us to obtain the full sequence of the plastid genome of $R$. phalloides.

The sequencing coverage in the $R$. phalloides plastid genome ranges from approximately $3,000 \times$ in the least AT-rich regions to 17 in the most AT-rich regions (Fig. S1). The AT content and the sequencing coverage correlate with a Spearman's correlation coefficient of -0.93 . Read insert size also depends on the AT content, with the least AT-rich regions covered by reads with an insert size of approximately $300 \mathrm{bp}$ and the most AT-rich regions with an insert size of approximately 200 bp (Fig. S2); Spearman's correlation coefficient was -0.69 . We suppose that the coverage drop associated with high AT content could be the reason why the authors of a work dedicated to an analysis of the Lophophytum mirabile (also a completely heterotrophic plant from the same family as $R$. phalloides) did not observe contigs with plastid genes (Sanchez-Puerta et al., 2017). Additionally, in R. lagascae, which was reported to have no plastid genome (Molina et al., 2014), it may potentially be present but be unnoticed due to its high AT content. Rafflesia lagascae genome assembly was performed using approximately 400 million Illumina reads, the same amount we used for the assembly of $R$. phalloides. Therefore, if $R$. lagascae indeed possesses a plastid genome, it should be much more AT-rich than the plastid genome of $R$. phalloides.

The AT content is high in protein-coding genes (the average value weighted by length is $88.1 \%$ ), as well as ncRNA-coding genes (the average value weighted by length is $77.5 \%$ ) and non-coding regions (the average value weighted by length is $93.8 \%$ ). In protein-coding genes, this led not only to a shift in codon frequencies towards AT-rich codons (Table S4) but also to a shift in amino acid frequencies in proteins, with amino acids encoded by AT-rich codons used more (Fig. 3, Table S5). For example, isoleucine, the amino acid with the most AT-rich codons, is used two times more often in the proteins encoded in the plastid genome of $R$. phalloides than in homologous proteins of phylogenetically close mixotrophic species. Similarly, glycine, whose codons are among the most GC-rich, is used two times more rarely. Plastid sequences of Balanophora experience the same effects. Additionally, the genetic code in the plastid genomes of Balanophora is non-canonical, utilising TAG (which is a stop codon in most genetic codes) as the tryptophan codon instead of the typical TGG. In contrast, the plastid genome of $R$. phalloides uses TGG for tryptophan, whereas the TAG codon is not used at all, even as a stop codon.

Interestingly, such high AT content has led to convergence of gene sequences of R. phalloides with sequences from phylogenetically distant AT-rich species. When aligning sequences of genes and proteins of $R$. phalloides to sequences from NCBI NT and NR databases, respectively,

PeerJ reviewing PDF | (2018:12:33547:2:0:ACCEPTED 8 Jul 2019) 
458

459

460

461

462

463

464

465

466

467

468

469

470

471

472

473

474

475

476

477

478

479

480

481

482

483

484

485

486

487

488

489

490

491

492

493

494

495

496

497

498

499

the best matches are often sequences from distantly related heterotrophic plants whose plastid genomes also have high AT content (Table S6). There are also many matches to sequences from various protists and some matches to sequences of animals and bacteria. Not all the matches are to homologous sequences, with some resulting from accidental similarity to non-coding sequences.

We thoroughly investigated one of the prominent cases of convergence - the rrn 16 gene. This particular gene was selected for the analysis because it is the only gene whose sequences are known for 3 genera of Balanophoraceae (Rhopalocnemis, Corynaea, Balanophora). This allowed us to check whether the convergence with distant species could also be observed in other genera of Balanophoraceae. BLASTN alignment of $r r n 16$ of $R$. phalloides to NCBI NT produces two best hits to other species of Balanophoraceae, namely C. crassa and B. japonica (for which only this plastid gene is sequenced and available in GenBank), whereas the next several dozen matches were to protists from the genera Plasmodium, Nitzschia and Leucocytozoon, belonging to SAR. Our initial hypothesis was a horizontal transfer from SAR to a common ancestor of the aforementioned Balanophoraceae. This was supported by the fact that a phylogenetic analysis of rrn 16 places the sequences of Balanophoraceae within SAR with very high bootstrap support (Fig. 4). A simple counterargument is that Plasmodium, Nitzschia and Leucocytozoon, though all belonging to SAR, are, in fact, quite distant phylogenetically from each other (with Nitzschia belonging to Stramenopiles, and Plasmodium and Leucocytozoon to Alveolata), and thus the fact that they appear in BLAST results together suggests some sort of bias. What is common for the species of the genera whose rrn 16 produces best matches to rrn16 of $R$. phalloides is that they have extremely high AT content, close to that of $R$. phalloides. Among the BLAST matches, the best 20 matching sequences of SAR have an AT content ranging from $62.6 \%$ to $77.2 \%$, with the average value $75.1 \%$. The AT content of the rrnl6 of $R$. phalloides is $77.2 \%$. For comparison, AT contents of rrn 16 from close mixotrophic relatives of $R$. phalloides, namely $V$. album, $O$. alba, C. manillana and S. jasminodora, have values typical for autotrophic plants ranging from $43.3 \%$ to $43.5 \%$. This led us to guess that the similarity originates not from the phylogenetic relatedness of rrn16 of $R$. phalloides to rrn16 of Plasmodium, Nitzschia and Leucocytozoon but from convergence because of their high AT content.

To test whether the grouping of rrn16 from Balanophoraceae with SAR was a consequence of the high AT content, we removed from the multiple alignment of rrn 16 all columns that contained adenines or thymines in R. phalloides, C. crassa or B. japonica and rebuilt the tree three times using these three produced alignments. One may expect that if the grouping was a consequence of the high AT content, the removal of columns with adenines or thymines would lead to a relocation of the respective species from SAR. When this operation was performed for $R$. phalloides (Fig. S3), it moved to a poorly resolved group containing all non-Balanophoraceae Embryophyta and also several representatives of SAR, while C. crassa and B. japonica remained in the same group as in Fig. 4. When the same procedure was performed for C. crassa, C. crassa moved to Embryophyta, while $R$. phalloides and B. japonica did not move (Fig. S4). Similarly, after this operation was done for $B$. japonica, it moved to a group that contained all Embryophyta plus Paulinella micropora (a species of SAR), while R. phalloides and C. crassa did not (Fig. $\mathrm{S} 5)$. Overall, although the trees are mostly poorly resolved, the fact that the removal of columns 
500 with adenines and thymines from the multiple alignment moves a species of Balanophoraceae 501 towards Embryophyta suggests that this similarity with SAR was a consequence of AT-richness.

502 An alternative explanation for the seeming phylogenetic closeness of $r r n 16$ of these three species

503 of Balanophoraceae to rrn16 of SAR can be long branch attraction, but it is a characteristic

504

505 problem of the maximum parsimony method and it affects phylogenetic trees built with the maximum likelihood method to a lesser degree (Kück et al., 2012). Additionally, the similarity of rrn 16 orthologues can potentially be a result of misalignment, but the alignment was good, and the convergence was clearly observed in the alignment (Supplemental Data S1-S2).

Overall, our results suggest that phylogenetic analyses of heterotrophic plants (and, in general, of any species whose genomes have highly biased nucleotide composition) should be performed cautiously, as even bootstrap support values of 100 do not guarantee reliable phylogenetic reconstruction in such cases.

Natural selection and substitution rate in the plastid genome of $R$.

515

The nucleotide substitution rate is known to be increased in plastid genomes of heterotrophic plants, ranging from a hardly detectable increase in plants that have lost their photosynthetic ability recently (Barrett, Wicke \& Sass, 2018) to a nearly 100-fold increase with respect to the closest photosynthetic species in the most reduced plastid genomes (Bellot \& Renner, 2015). To the best of our knowledge, the reason for this increase is not yet known (and will be discussed in more details in the section "Why is the AT content so high?").

To compare the substitution rate in $R$. phalloides with the rates in its closest mixotrophic relatives, one should first determine the phylogenetic placement of $R$. phalloides relative to the species of comparison. The placement of the family Balanophoraceae has long been debated, with some scientists stating that it does not even belong to Santalales (Kuijt, 1968; Cronquist, 1981; Takhtadzhian, 2009). A recent work, which utilised sequences of 7 genes for phylogeny evaluation, suggested that Balanophoraceae indeed belong to Santalales (Su et al., 2015). Moreover, the results of that work suggest polyphyly of Balanophoraceae, which consist of two clades: "Balanophoraceae A" and "Balanophoraceae B". A common feature of Balanophoraceae $A$ is that they have highly increased substitution rates, and a common feature of Balanophoraceae B is that their substitution rates are approximately the same as in autotrophic and mixotrophic Santalales. Although it analysed 11 species of Balanophoraceae, that study did not analyse $R$. phalloides. To estimate the phylogenetic relationships of $R$. phalloides, we added the sequences of its nuclear 18S rDNA and 26S rDNA to the alignment of sequences from 186 species used in that article and rebuilt the tree. As one may have expected, $R$. phalloides is placed in Balanophoraceae A, with a bootstrap support value of 100 (Fig. S6). It is sister to a group of C. crassa and Helosis cayennensis.

538 To evaluate substitution rates, $\mathrm{dN}$ and $\mathrm{dS}$ in the plastid genome of $R$. phalloides, we used 
540 plastid genomes of several other species of Santalales, available as of 2017. The genes $y c f 1, y c f 2$ 541 and $r p s 7$ were excluded from the analysis because their sequences in $R$. phalloides could not be 542 reliably aligned with homologous sequences of other species owing to the high amount of accumulated mutations. The analysis by PAML showed that the number of nucleotide

544

545

546

547

548

549

550

551

552

553

554

555

556

557

558

559

560

561

562

563

564

565

566

567

568

569

570

571

572

573

574

575

576

577

578

579

580
1. The model of nucleotide substitutions used in PAML utilises the equilibrium codon frequencies, equal for all branches. This is definitely not the case in the studied Santalales, as the codon frequencies in the plastid genome of $R$. phalloides highly differ from those in plastid genomes of mixotrophic Santalales (Table S4).

We are aware of a single tool for phylogenetic analyses that can take into account different codon frequencies in different sequences. This is a program collection BppSuite. However, the analysis of these data by BppSuite provided a value of approximately 44,000 instead of 21 , which was probably owing to an algorithmic mistake.

2. Non-synonymous substitutions quickly reach saturation, and thus the number of nonsynonymous substitutions is underestimated for long branches (dos Reis \& Yang, 2013). The same is true for synonymous substitutions (Vanneste, Van de Peer \& Maere, 2013).

3. We removed columns in the alignment with many differences between species using the program Gblocks, because such columns may result from misalignment. As regions of genes with positive or weak negative selection accumulate mutations faster, such regions can also be potentially removed by Gblocks, leading to underestimation of substitution rates.

4. We failed to produce reliable alignments for the genes ycf1, ycf2 and rps7, consequently the substitution rate in these genes may be higher than in others. Therefore, the exclusion of these genes from the analysis may lead to underestimation of the true substitution rate.

The substitution rate analysis for $B$. reflexa provided a very similar result to that of $R$. phalloides. The $\mathrm{dN} / \mathrm{dS}$ values on the branches of Balanophoraceae were slightly lower than on the branches of mixotrophic Santalales. Because the estimation of the $\mathrm{dN}$ and $\mathrm{dS}$ values could be imprecise, the values of $\mathrm{dN} / \mathrm{dS}$ should also be treated cautiously. In the future, the problems associated with the analysis of long branches can be reduced by increasing the taxon sampling for Balanophoraceae, thus decreasing the branch lengths. Although the precise value of $\mathrm{dN} / \mathrm{dS}$ on the branch of $R$. phalloides is hard to estimate, the selection acting on its genes is definitely nonneutral, as open reading frames of all the genes are intact. If we denote the probability that there is a specific codon in a specific position as $\mathrm{P}(\mathrm{X})$, and the AT content of a gene as $\alpha$, then the probability that a random codon is a stop is

$\mathrm{P}($ Stop $)=\mathrm{P}(\mathrm{TAA})+\mathrm{P}(\mathrm{TGA})+\mathrm{P}(\mathrm{TAG})=(\alpha / 2) \times(\alpha / 2) \times(\alpha / 2)+(\alpha / 2) \times((1-\alpha) / 2) \times(\alpha / 2)+(\alpha / 2) \times(\alpha / 2) \times((1-$ $\alpha) / 2)=\alpha^{2} / 4-\alpha^{3} / 8$.

As the weighted (by length) average AT content in protein-coding genes of $R$. phalloides is $88 \%$, the probability of a random codon being a stop, as follows from this equation, is approximately

Peer] reviewing PDF | (2018:12:33547:2:0:ACCEPTED 8 Jul 2019) 
$58111 \%$. This means that because stop codons are AT-rich, in a random sequence with such a high

582 AT content as in $R$. phalloides, every 9 th codon will be a stop. Therefore, a strong negative

583 selection must be acting on the genes to keep open reading frames unbroken.

584

585 Other genomes of $R$. phalloides

586

587

588

Sequencing of approximately 400 million paired-end reads could have been enough to assemble

589 the mitochondrial and the nuclear genomes of $R$. phalloides. The alignment by BLASTN and

590 TBLASTN of ncRNAs and proteins, respectively, encoded in mitochondrial genomes of the reference species to the contigs of $R$. phalloides revealed several dozen matching contigs with coverages of approximately 5,000× and lengths of approximately 1,000-5,000 bp. They are probably short mitochondrial chromosomes, similar to those observed in the plant L. mirabile (Sanchez-Puerta et al., 2017), also from Balanophoraceae, whose mitochondrial genome putatively consists of 54 small circular chromosomes. We are ready to provide the mitochondrial contigs upon request.

596 Known sizes of nuclear genomes of plants from Santalales vary from approximately $200 \mathrm{Mbp}$ in Santalum album (Mahesh et al., 2018) to approximately $100 \mathrm{Gbp}$ in Viscum album (Zonneveld, 2010). For example, if the nuclear genome size in $R$. phalloides is $500 \mathrm{Mbp}, 400$ million 150-bplong reads will produce a coverage of approximately $400 \times 150 / 500=120 \times$, which is enough for a

600 draft assembly. To estimate the nuclear genome size, we built a k-mer frequency histogram (Fig. S7). The peak of the distribution, corresponding to the k-mer coverage of the nuclear genome,

602

603

604 was difficult to determine, but it was below the k-mer coverage value of $2 \times$. As the k-mer size (21) was much lower than the read size (150), the read coverage was approximately equal to the $\mathrm{k}$-mer coverage. Therefore, the nuclear genome size could be estimated to be at least

605 $400 \times 150 / 2=30,000 \mathrm{Mbp}$. Potentially, the genome size could be overestimated if there is a lot of contamination (for example by DNA of endophytic bacteria and fungi), but a taxonomic analysis of reads suggests that contamination in unlikely to be high (Table S7). The assembly of a 30,000Mbp-long genome is impossible using only the reads produced in the current study. Instead of the complete nuclear genome assembly, we plan to study it by means of transcriptome assembly, which is the subject of our next work.

611

\section{Why is the AT content so high?}

613

614 The increase in the AT content in the plastid genomes of heterotrophic plants, as well as the 615 increase in their substitution rates, are known and much-discussed phenomena (Bromham, 616 Cowman \& Lanfear, 2013; Wicke et al., 2016; Hadariová et al., 2018; Wicke \& Naumann, 617 2018). However, their origin is still unknown. The simplest hypothesis for the increase in the 618 substitution rate could be the relaxation of selection acting on genes. However, plastid genes of 619 heterotrophic plants usually show no signs of relaxed selection, except for photosynthesis-related 
620 genes during pseudogenization. Interestingly, a high AT content and substitution rate have also 621 been observed in plastids of non-photosynthetic protists (such as Plasmodium) (Oborník et al., 622 2009), which lost the genes required for photosynthesis after the transition to a heterotrophic 623 lifestyle. Additionally, both of these phenomena have been observed in genomes of 624 endosymbiotic bacteria (McCutcheon \& Moran, 2011), which may be dozens of times shorter 625 than genomes of their free-living relatives owing to the loss of genes required, for example, for 626 biosynthesis of substances that are now provided to the symbiont by its host. Therefore, these 627 two phenomena are probably not only unrestricted to plants but are not even related to the loss of 628 photosynthesis.

629 A phenomenon that can simultaneously result in both an increase of AT content and an increase 630 of substitution rate is the reduction in genome recombination intensity. A plastid genome is 631 capable of recombining both within itself (the recombination of two copies of the inverted 632 repeat) (Zhu et al., 2016; Li et al., 2016) and between two copies of a genome (Maréchal \& 633 Brisson, 2010). The recombination is an important step in repair, both in plastids (Zampini et al., 634 2017) and in bacteria (Cox, 1998), so the reduction in recombination will increase the substitution rate. Also, gene conversion in plastid (Wu \& Chaw, 2015; Zhitao Niu et al., 2017) as well as in bacterial (Lassalle et al., 2015) genomes is GC-biased, although earlier gene conversion in plastid genomes was supposed to be AT-biased (Khakhlova \& Bock, 2006). This means that if there is a mismatch between an adenine or a thymine on one strand versus a guanine or a cytosine on the other during a recombination, it is more likely that the guanine or the cytosine will be kept, while the adenine or the thymine will be removed and replaced by a cytosine or a guanine, which is complementary to the base on the other strand. Therefore, recombination aids in increasing the GC content in plastid and bacterial genomes, and a decrease in recombination will make a genome more AT-rich. The link between the low recombination rate and the high AT content has already been proposed for endosymbiotic bacteria with small genomes (Lassalle et al., 2015).

Recently, it was shown that in transcriptomes of the heterotrophic plants Epipogium aphyllum, E. roseum and Hypopitys monotropa, the transcript of the protein RECA1, which is required for recombination of the plastid genomes, is absent (Schelkunov, Penin \& Logacheva, 2018). This may support the above hypothesis. However, the direct reason for the loss of RECA1 is not known. A potential explanation for the loss could be that during the transition from a mixotrophic to a heterotrophic lifestyle, plastid enzymes related to photosynthesis accumulate mutations, and since a mutated enzyme may be harmful for the organism, it is evolutionarily adaptive to accumulate the mutations very fast to quickly achieve complete disruption of a gene, instead of having a semi-degraded gene encoding a harmful protein. This effect, consisting of elimination of pseudogenes at rates faster than neutral, has already been shown to take place in bacteria (Kuo \& Ochman, 2010). Therefore, in the period directly following the loss of photosynthesis, it may be beneficial for the plant to disturb the plastid recombination and thus disturb the repair. In fact, this process may start even before the loss of photosynthesis, because in plastid genomes of mixotrophic plants $n d h$ genes often undergo pseudogenization (Wicke et al., 2011), and their quick removal may require an increased mutation accumulation rate. Such an increase in the mutation accumulation rate may require pseudogenization of genes of DNA 
662 replication, recombination and repair (DNA-RRR), such as RECA1, and once they are

663 pseudogenised, it will be hard for a plant to return to the normal repair intensity in the plastid

664 genome, making the transition to high mutation accumulation rates irreversible.

665 It is known that the mutation accumulation rate in heterotrophic plants (Bromham, Cowman \&

666 Lanfear, 2013), including Balanophoraceae (Su \& Hu, 2012; Su et al., 2015), is also increased in 667 nuclear and mitochondrial genomes, although to a lesser extent than in plastid genomes. These 668 phenomena are also still unexplained. The nuclear genome contains more than a hundred 669 (Schelkunov, Penin \& Logacheva, 2018) genes that encode proteins, working in multisubunit 670 complexes with proteins, encoded in the plastid genome. These are the genes encoding proteins

671 of the electron-transfer chain, the plastid-encoded RNA polymerase (PEP), the plastid ribosome

672 and others. When a species loses its photosynthetic ability, the nuclear-encoded genes of the

673 electron-transfer chain are no longer under selective pressure and start to accumulate mutations.

674 Therefore, their proteins may become harmful and may require quick elimination. Thus, the

675 increase in the nuclear mutation accumulation rate, which may speed up the accumulation of

676 disruptive mutations in these genes, may also by selectively beneficial. The increase in the

677 mutation accumulation rate in the mitochondrial genome could potentially be explained by the

678 fact that many DNA-RRR proteins are common for the plastid and the mitochondrial genomes

679 (Shedge et al., 2007; Carrie \& Small, 2013). Therefore, if it is selectively beneficial to increase

680 the mutation accumulation rate in the plastid genome, the mitochondrial genome may also be

681 affected.

682 This hypothesis of accelerated junk removal may be tested by studying plastid and nuclear 683 genomes of many related heterotrophic species and checking whether the crumbling genes 684 accumulate mutations at rates faster than neutral shortly after the loss of photosynthesis and 685 whether some of the DNA-RRR genes deteriorate at the same time.

686

687 Conclusions and future studies

688 The plastid genome of $R$. phalloides profoundly differs from plastid genomes of typical plants, 689 including the massive gene loss, the increased substitution rate and the high AT content. By 690 decreasing sequencing coverage, such high AT content may "hide" plastid genomes of some

691 heterotrophic plants, making these genomes harder to find by means of high-throughput 692 sequencing. Alterations in the nuclear genome, accompanying these changes in the plastid 693 genome, are an interesting issue. Our next work will be dedicated to the study of the nuclear 694 genome of $R$. phalloides by means of transcriptome sequencing.

695

\section{Acknowledgements}

697 We are grateful to Alina Alexandrova for field assistance. We would also like to thank three

698 reviewers: Sean Graham, Jeff Palmer and one anonymous reviewer for their valuable

699 suggestions. 


\section{References}

702 Abascal F, Zardoya R, Telford MJ. 2010. TranslatorX: multiple alignment of nucleotide

703

704

705

706

707

708

709

710

711

712

713

714

715

716

717

718

719

720

721

722

723

724 sequences guided by amino acid translations. Nucleic Acids Research 38:W7-W13. DOI: 10.1093/nar/gkq291.

Bankevich A, Nurk S, Antipov D, Gurevich AA, Dvorkin M, Kulikov AS, Lesin VM, Nikolenko SI, Pham S, Prjibelski AD, Pyshkin AV, Sirotkin AV, Vyahhi N, Tesler G, Alekseyev MA, Pevzner PA. 2012. SPAdes: a new genome assembly algorithm and its applications to single-cell sequencing. Journal of Computational Biology 19:455-477. DOI: $10.1089 / \mathrm{cmb} .2012 .0021$.

Barbrook AC, Howe CJ, Purton S. 2006. Why are plastid genomes retained in nonphotosynthetic organisms? Trends in Plant Science 11:101-108. DOI: 10.1016/j.tplants.2005.12.004.

Barrett CF, Freudenstein JV, Li J, Mayfield-Jones DR, Perez L, Pires JC, Santos C. 2014. Investigating the path of plastid genome degradation in an early-transitional clade of heterotrophic orchids, and implications for heterotrophic angiosperms. Molecular Biology and Evolution 31:3095-3112. DOI: 10.1093/molbev/msu252.

Barrett CF, Wicke S, Sass C. 2018. Dense infraspecific sampling reveals rapid and independent trajectories of plastome degradation in a heterotrophic orchid complex. New Phytologist 218:1192-1204. DOI: 10.1111/nph.15072.

Bellot S, Renner SS. 2015. The plastomes of two species in the endoparasite genus Pilostyles (Apodanthaceae) each retain just five or six possibly functional genes. Genome Biology and Evolution:evv251. DOI: 10.1093/gbe/evv251.

Benjamini Y, Speed TP. 2012. Summarizing and correcting the GC content bias in highthroughput sequencing. Nucleic Acids Research 40:e72. DOI: 10.1093/nar/gks001. 
725 Bolger AM, Lohse M, Usadel B. 2014. Trimmomatic: a flexible trimmer for Illumina sequence 726 data. Bioinformatics 30:2114-2120. DOI: 10.1093/bioinformatics/btu170.

727 Bouchier C, Ma L, Créno S, Dujon B, Fairhead C. 2009. Complete mitochondrial genome 728 sequences of three Nakaseomyces species reveal invasion by palindromic GC clusters 729 and considerable size expansion. FEMS yeast research 9:1283-1292. DOI: 10.1111/j.1567-1364.2009.00551.x.

731 Bromham L, Cowman PF, Lanfear R. 2013. Parasitic plants have increased rates of molecular evolution across all three genomes. BMC evolutionary biology 13:126.

733 Camacho C, Coulouris G, Avagyan V, Ma N, Papadopoulos J, Bealer K, Madden TL. 2009. BLAST+: architecture and applications. BMC Bioinformatics 10:421. DOI: $10.1186 / 1471-2105-10-421$.

736

737

738

739

740

741

742

743

744

745

746

Carrie C, Small I. 2013. A reevaluation of dual-targeting of proteins to mitochondria and chloroplasts. Biochimica et Biophysica Acta (BBA) - Molecular Cell Research 1833:253259. DOI: 10.1016/j.bbamcr.2012.05.029.

Castresana J. 2000. Selection of conserved blocks from multiple alignments for their use in phylogenetic analysis. Molecular Biology and Evolution 17:540-552.

Cox MM. 1998. A broadening view of recombinational DNA repair in bacteria. Genes to Cells 3:65-78. DOI: 10.1046/j.1365-2443.1998.00175.x.

Cronquist A. 1981. An integrated system of classification of flowering plants. New York: Columbia University Press.

Cummings MP, Welschmeyer NA. 1998. Pigment composition of putatively achlorophyllous angiosperms. Plant Systematics and Evolution 210:105-111. DOI: 10.1007/BF00984730. 
747 Daniell H, Lin C-S, Yu M, Chang W-J. 2016. Chloroplast genomes: diversity, evolution, and

748

749

750

751

752

753

754

755

756

757

758

759

760

761

762

763

764

765

766

767 applications in genetic engineering. Genome Biology 17. DOI: 10.1186/s13059-0161004-2.

Doyle J. 1987. A rapid DNA isolation procedure for small quantities of fresh leaf tissue. Phytochem Bull 19:11-15.

Edgar RC. 2004. MUSCLE: multiple sequence alignment with high accuracy and high throughput. Nucleic Acids Research 32:1792-1797. DOI: 10.1093/nar/gkh340.

Finn RD, Attwood TK, Babbitt PC, Bateman A, Bork P, Bridge AJ, Chang H-Y, Dosztányi Z, El-Gebali S, Fraser M, Gough J, Haft D, Holliday GL, Huang H, Huang X, Letunic I, Lopez R, Lu S, Marchler-Bauer A, Mi H, Mistry J, Natale DA, Necci M, Nuka G, Orengo CA, Park Y, Pesseat S, Piovesan D, Potter SC, Rawlings ND, Redaschi N, Richardson L, Rivoire C, Sangrador-Vegas A, Sigrist C, Sillitoe I, Smithers B, Squizzato S, Sutton G, Thanki N, Thomas PD, Tosatto SCE, Wu CH, Xenarios I, Yeh L-S, Young S-Y, Mitchell AL. 2017. InterPro in 2017-beyond protein family and domain annotations. Nucleic Acids Research 45:D190-D199. DOI: 10.1093/nar/gkw1107.

Graham SW, Lam VKY, Merckx VSFT. 2017. Plastomes on the edge: the evolutionary breakdown of mycoheterotroph plastid genomes. New Phytologist 214:48-55. DOI: 10.1111/nph.14398.

Gualberto JM, Newton KJ. 2017. Plant mitochondrial genomes: dynamics and mechanisms of mutation. Annual Review of Plant Biology 68:225-252. DOI: 10.1146/annurev-arplant043015-112232. 
768 Guéguen L, Duret L. 2017. Unbiased estimate of synonymous and non-synonymous substitution 769 rates with non-stationary base composition. Molecular Biology and Evolution. DOI:

770 10.1093/molbev/msx308.

771

772

773

Guéguen L, Gaillard S, Boussau B, Gouy M, Groussin M, Rochette NC, Bigot T, Fournier D, Pouyet F, Cahais V, Bernard A, Scornavacca C, Nabholz B, Haudry A, Dachary L, Galtier N, Belkhir K, Dutheil JY. 2013. Bio++: efficient extensible libraries and tools for computational molecular evolution. Molecular Biology and Evolution 30:1745-1750. DOI: $10.1093 / \mathrm{molbev} / \mathrm{mst} 097$.

Hadariová L, Vesteg M, Hampl V, Krajčovič J. 2018. Reductive evolution of chloroplasts in non-photosynthetic plants, algae and protists. Current Genetics 64:365-387. DOI: 10.1007/s00294-017-0761-0.

Howe CJ, Smith A. 1991. Plants without chlorophyll. Nature 349:109-109. DOI: $10.1038 / 349109 \mathrm{c} 0$.

Jones P, Binns D, Chang H-Y, Fraser M, Li W, McAnulla C, McWilliam H, Maslen J, Mitchell A, Nuka G, Pesseat S, Quinn AF, Sangrador-Vegas A, Scheremetjew M, Yong S-Y, Lopez R, Hunter S. 2014. InterProScan 5: genome-scale protein function classification. Bioinformatics 30:1236-1240. DOI: 10.1093/bioinformatics/btu031.

Katoh K, Standley DM. 2013. MAFFT multiple sequence alignment software version 7: improvements in performance and usability. Molecular Biology and Evolution 30:772780. DOI: $10.1093 / \mathrm{molbev} / \mathrm{mst} 010$.

Khakhlova O, Bock R. 2006. Elimination of deleterious mutations in plastid genomes by gene conversion. The Plant Journal 46:85-94. DOI: 10.1111/j.1365-313X.2006.02673.x. 
790 Krause K. 2015. Grand-scale theft: Kleptoplasty in parasitic plants? Trends in Plant Science $791 \quad 20: 196-198$. DOI: $10.1016 / j . t p l a n t s .2015 .03 .005$.

792 Kück P, Mayer C, Wägele J-W, Misof B. 2012. Long Branch Effects Distort Maximum 793 Likelihood Phylogenies in Simulations Despite Selection of the Correct Model. PLoS 794 ONE 7:e36593. DOI: 10.1371/journal.pone.0036593.

795 Kuijt J. 1968. Mutual Affinities of Santalalean Families. Brittonia 20:136. DOI: $10.2307 / 2805616$.

Kuo C-H, Ochman H. 2010. The Extinction Dynamics of Bacterial Pseudogenes. PLoS Genetics Kumar AM, Schaub U, Söll D, Ujwal ML. 1996. Glutamyl-transfer RNA: at the crossroad between chlorophyll and protein biosynthesis. Trends in Plant Science 1:371-376. DOI: 10.1016/S1360-1385(96)80311-6. 6:e1001050. DOI: 10.1371/journal.pgen.1001050.

Lagesen K, Hallin P, Rødland EA, Staerfeldt H-H, Rognes T, Ussery DW. 2007. RNAmmer: consistent and rapid annotation of ribosomal RNA genes. Nucleic Acids Research

Lassalle F, Périan S, Bataillon T, Nesme X, Duret L, Daubin V. 2015. GC-Content Evolution in 810 Bacterial Genomes: The Biased Gene Conversion Hypothesis Expands. PLOS Genetics 811 35:3100-3108. DOI: 10.1093/nar/gkm160.

Lam VKY, Soto Gomez M, Graham SW. 2015. The highly reduced plastome of mycoheterotrophic Sciaphila (Triuridaceae) is colinear with its green relatives and is under strong purifying selection. Genome Biology and Evolution 7:2220-2236. DOI: 10.1093/gbe/evv134. 11:e1004941. DOI: 10.1371/journal.pgen.1004941. 
812 Li F-W, Kuo L-Y, Pryer KM, Rothfels CJ. 2016. Genes Translocated into the Plastid Inverted

813 Repeat Show Decelerated Substitution Rates and Elevated GC Content. Genome Biology

814 and Evolution 8:2452-2458. DOI: 10.1093/gbe/evw167.

815 Liu T-J, Zhang C-Y, Yan H-F, Zhang L, Ge X-J, Hao G. 2016. Complete plastid genome 816 sequence of Primula sinensis (Primulaceae): structure comparison, sequence variation 817 and evidence for $a c c D$ transfer to nucleus. PeerJ 4:e2101. DOI: 10.7717/peerj.2101.

818 Logacheva MD, Schelkunov MI, Penin AA. 2011. Sequencing and analysis of plastid genome in 819 mycoheterotrophic orchid Neottia nidus-avis. Genome Biology and Evolution 3:1296820 1303. DOI: $10.1093 /$ gbe/evr102.

821 Lowe TM, Eddy SR. 1997. tRNAscan-SE: a program for improved detection of transfer RNA 822 genes in genomic sequence. Nucleic Acids Research 25:955-964.

823 Mahesh HB, Subba P, Advani J, Shirke MD, Loganathan RM, Chandana SL, Shilpa S, 824 Chatterjee O, Pinto SM, Prasad TSK, Gowda M. 2018. Multi-Omics Driven Assembly 825 and Annotation of the Sandalwood ( Santalum album ) Genome. Plant Physiology 176:2772-2788. DOI: 10.1104/pp.17.01764.

827 Marçais G, Kingsford C. 2011. A fast, lock-free approach for efficient parallel counting of 828 occurrences of k-mers. Bioinformatics 27:764-770. DOI: 10.1093/bioinformatics/btr011.

829 Maréchal A, Brisson N. 2010. Recombination and the maintenance of plant organelle genome 830 stability. New Phytologist 186:299-317. DOI: 10.1111/j.1469-8137.2010.03195.x.

831 McCutcheon JP, Moran NA. 2011. Extreme genome reduction in symbiotic bacteria. Nature 832 Reviews Microbiology 10:13-26. DOI: 10.1038/nrmicro2670. 
833 McKain MR, Hartsock RH, Wohl MM, Kellogg EA. 2017. Verdant: automated annotation,

834 alignment and phylogenetic analysis of whole chloroplast genomes. Bioinformatics

835 33:130-132. DOI: 10.1093/bioinformatics/btw583.

836 Merckx VSFT, Freudenstein JV, Kissling J, Christenhusz MJM, Stotler RE, Crandall-Stotler B,

837 Wickett N, Rudall PJ, Maas-van de Kamer H, Maas PJM. 2013. Taxonomy and

838 Classification. In: Merckx V ed. Mycoheterotrophy. New York, NY: Springer New York, 839 19-101. DOI: 10.1007/978-1-4614-5209-6_2.

840 Millen RS, Olmstead RG, Adams KL, Palmer JD, Lao NT, Heggie L, Kavanagh TA, Hibberd 841 JM, Gray JC, Morden CW, Calie PJ, Jermiin LS, Wolfe KH. 2001. Many parallel losses 842 of infA from chloroplast DNA during angiosperm evolution with multiple independent

844 Molina J, Hazzouri KM, Nickrent D, Geisler M, Meyer RS, Pentony MM, Flowers JM, Pelser P, Barcelona J, Inovejas SA, Uy I, Yuan W, Wilkins O, Michel C-I, LockLear S, Concepcion GP, Purugganan MD. 2014. Possible loss of the chloroplast genome in the parasitic flowering plant Rafflesia lagascae (Rafflesiaceae). Molecular Biology and Evolution 31:793-803. DOI: 10.1093/molbev/msu051.

Naumann J, Der JP, Wafula EK, Jones SS, Wagner ST, Honaas LA, Ralph PE, Bolin JF, Maass E, Neinhuis C, Wanke S, dePamphilis CW. 2016. Detecting and characterizing the highly divergent plastid genome of the nonphotosynthetic parasitic plant Hydnora visseri (Hydnoraceae). Genome Biology and Evolution 8:345-363. DOI: 10.1093/gbe/evv256.

853 Nawrocki EP, Burge SW, Bateman A, Daub J, Eberhardt RY, Eddy SR, Floden EW, Gardner 854 PP, Jones TA, Tate J, Finn RD. 2015. Rfam 12.0: updates to the RNA families database. Nucleic Acids Research 43:D130-D137. DOI: 10.1093/nar/gku1063. 
856 Nawrocki EP, Eddy SR. 2013. Infernal 1.1: 100-fold faster RNA homology searches.

857 Bioinformatics 29:2933-2935. DOI: 10.1093/bioinformatics/btt509.

858 Oborník M, Janouškovec J, Chrudimský T, Lukeš J. 2009. Evolution of the apicoplast and its

859 hosts: From heterotrophy to autotrophy and back again. International Journal for

$860 \quad$ Parasitology 39:1-12. DOI: 10.1016/j.ijpara.2008.07.010.

861 Peden JF. 1999. Analysis of codon usage. PhD Thesis Thesis. UK: University of Nottingham.

862 dos Reis M, Yang Z. 2013. Why Do More Divergent Sequences Produce Smaller

863 Nonsynonymous/Synonymous Rate Ratios in Pairwise Sequence Comparisons? Genetics

864 195:195-204. DOI: 10.1534/genetics.113.152025.

865 Rousseau-Gueutin M, Huang X, Higginson E, Ayliffe M, Day A, Timmis JN. 2013. Potential

866 functional replacement of the plastidic acetyl-CoA carboxylase subunit (accD) gene by

867 recent transfers to the nucleus in some angiosperm lineages. Plant Physiology 161:1918-

868 1929. DOI: $10.1104 /$ pp.113.214528.

869 Sakamoto W, Takami T. 2018. Chloroplast DNA Dynamics: Copy Number, Quality Control and $870 \quad$ Degradation. Plant and Cell Physiology 59:1120-1127. DOI: 10.1093/pcp/pcy084.

871 Sanchez-Puerta MV, García LE, Wohlfeiler J, Ceriotti LF. 2017. Unparalleled replacement of 872

873 native mitochondrial genes by foreign homologs in a holoparasitic plant. New Phytologist

874 Schelkunov MI, Penin AA, Logacheva MD. 2018. RNA-seq highlights parallel and contrasting 875 patterns in the evolution of the nuclear genome of fully mycoheterotrophic plants. $B M C$ Genomics 19. DOI: 10.1186/s12864-018-4968-3.

877 Schelkunov MI, Shtratnikova VY, Nuraliev MS, Selosse M-A, Penin AA, Logacheva MD. 2015. 878 Exploring the limits for reduction of plastid genomes: a case study of the 

Biology and Evolution 7:1179-1191. DOI: 10.1093/gbe/evv019.

881 Seregin A. 2018. Moscow University Herbarium (MW). DOI: 10.15468/cpnhcc.

882 Shah N, Nute MG, Warnow T, Pop M. 2018. Misunderstood parameter of NCBI BLAST 883 impacts the correctness of bioinformatics workflows. Bioinformatics. DOI:

Shedge V, Arrieta-Montiel M, Christensen AC, Mackenzie SA. 2007. Plant Mitochondrial Recombination Surveillance Requires Unusual RecA and MutS Homologs. THE PLANT CELL ONLINE 19:1251-1264. DOI: 10.1105/tpc.106.048355.

Smith DR. 2012. Updating Our View of Organelle Genome Nucleotide Landscape. Frontiers in Genetics 3. DOI: 10.3389/fgene.2012.00175.

Smith DR, Lee RW. 2014. A plastid without a genome: evidence from the nonphotosynthetic green algal genus Polytomella. Plant physiology 164:1812-1819. DOI:

892 10.1104/pp.113.233718.

Stamatakis A. 2014. RAxML version 8: a tool for phylogenetic analysis and post-analysis of large phylogenies. Bioinformatics 30:1312-1313. DOI: 10.1093/bioinformatics/btu033.

Stöver BC, Müller KF. 2010. TreeGraph 2: Combining and visualizing evidence from different phylogenetic analyses. BMC Bioinformatics 11:7. DOI: 10.1186/1471-2105-11-7.

897 Su H-J, Barkman TJ, Hao W, Jones SS, Naumann J, Skippington E, Wafula EK, Hu J-M, Palmer JD, dePamphilis CW. 2019. Novel genetic code and record-setting AT-richness in the highly reduced plastid genome of the holoparasitic plant Balanophora. Proceedings of the National Academy of Sciences of the United States of America 116:934-943. DOI: 10.1073/pnas.1816822116. 
$902 \mathrm{Su} \mathrm{H}-\mathrm{J}, \mathrm{Hu}$ J-M. 2012. Rate heterogeneity in six protein-coding genes from the holoparasite

903 Balanophora (Balanophoraceae) and other taxa of Santalales. Annals of Botany

904 110:1137-1147. DOI: 10.1093/aob/mcs197.

905 Su H-J, Hu J-M, Anderson FE, Der JP, Nickrent DL. 2015. Phylogenetic relationships of

906 Santalales with insights into the origins of holoparasitic Balanophoraceae. Taxon 64:491-

907 506. DOI: $10.12705 / 643.2$.

908

Takhtadzhian AL. 2009. Flowering plants. New York: Springer.

909 Tiller N, Bock R. 2014. The translational apparatus of plastids and its role in plant development.

$910 \quad$ Molecular Plant 7:1105-1120. DOI: 10.1093/mp/ssu022.

911 Vanneste K, Van de Peer Y, Maere S. 2013. Inference of genome duplications from age

912 distributions revisited. Molecular Biology and Evolution 30:177-190. DOI:

$913 \quad 10.1093 / \mathrm{molbev} / \mathrm{mss} 214$.

914 Westwood JH, Yoder JI, Timko MP, dePamphilis CW. 2010. The evolution of parasitism in

915 plants. Trends in Plant Science 15:227-235. DOI: 10.1016/j.tplants.2010.01.004.

916 Wicke S, Müller KF, dePamphilis CW, Quandt D, Bellot S, Schneeweiss GM. 2016. Mechanistic

917 model of evolutionary rate variation en route to a nonphotosynthetic lifestyle in plants.

918 Proceedings of the National Academy of Sciences 113:9045-9050. DOI:

$919 \quad$ 10.1073/pnas.1607576113.

920 Wicke S, Naumann J. 2018. Molecular Evolution of Plastid Genomes in Parasitic Flowering

921 Plants. In: Advances in Botanical Research. Elsevier, 315-347. DOI:

$922 \quad$ 10.1016/bs.abr.2017.11.014. 
923 Wicke S, Schneeweiss GM, dePamphilis CW, Müller KF, Quandt D. 2011. The evolution of the 924 plastid chromosome in land plants: gene content, gene order, gene function. Plant 925 Molecular Biology 76:273-297. DOI: 10.1007/s11103-011-9762-4.

926 Wootton JC, Federhen S. 1993. Statistics of local complexity in amino acid sequences and 927 sequence databases. Computers \& Chemistry 17:149-163. DOI: 10.1016/0097-

928 8485(93)85006-X.

Wu C-S, Chaw S-M. 2015. Evolutionary Stasis in Cycad Plastomes and the First Case of 930 Plastome GC-Biased Gene Conversion. Genome Biology and Evolution 7:2000-2009.

931 DOI: $10.1093 /$ gbe/evv125.

932 933

Wyman SK, Jansen RK, Boore JL. 2004. Automatic annotation of organellar genomes with DOGMA. Bioinformatics 20:3252-3255. DOI: 10.1093/bioinformatics/bth352.

934 Yang Z. 2007. PAML 4: phylogenetic analysis by maximum likelihood. Molecular Biology and 935 Evolution 24:1586-1591. DOI: 10.1093/molbev/msm088.

936 Zampini É, Truche S, Lepage É, Tremblay-Belzile S, Brisson N. 2017. Plastid Genome Stability 937 938 and Repair. In: Li X-Q ed. Somatic Genome Variation in Animals, Plants, and

Zhitao Niu, Qingyun Xue, Hui Wang, Xuezhu Xie, Shuying Zhu, Wei Liu, Xiaoyu Ding. 2017. Mutational Biases and GC-Biased Gene Conversion Affect GC Content in the Plastomes of Dendrobium Genus. International Journal of Molecular Sciences 18:2307. DOI: 10.3390/ijms 18112307. 
944 Zhu A, Guo W, Gupta S, Fan W, Mower JP. 2016. Evolutionary dynamics of the plastid inverted 945 repeat: the effects of expansion, contraction, and loss on substitution rates. New $946 \quad$ Phytologist 209:1747-1756. DOI: 10.1111/nph.13743.

947 Zonneveld BJM. 2010. New Record Holders for Maximum Genome Size in Eudicots and 948 Monocots. Journal of Botany 2010:1-4. DOI: 10.1155/2010/527357. 
Figure 1

Map of the Rhopalocnemis phalloides plastid genome showing various features.

The circular-mapping plastid genome is represented linearly for convenience. Green arrows are rRNA-coding genes, red arrows are ribosomal protein-coding genes, and blue arrows are genes coding proteins with other functions. Grey arcs represent splicing. Blue columns show non-coding regions.

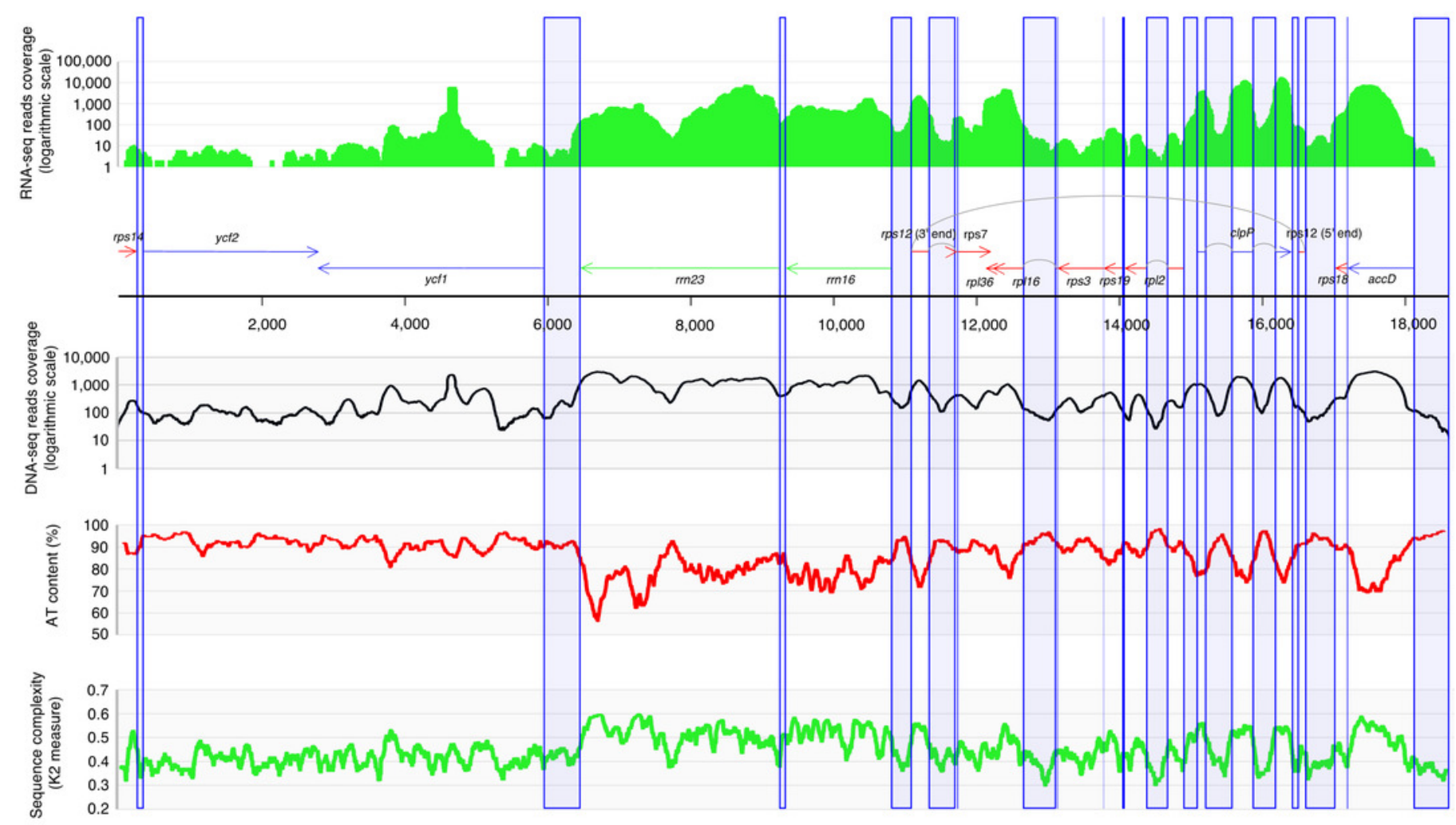


Figure 2

AT content and lengths of the plastid genomes of Embryophyta.

Red dots denote completely heterotrophic plants and black dots mixotrophic and completely autotrophic.

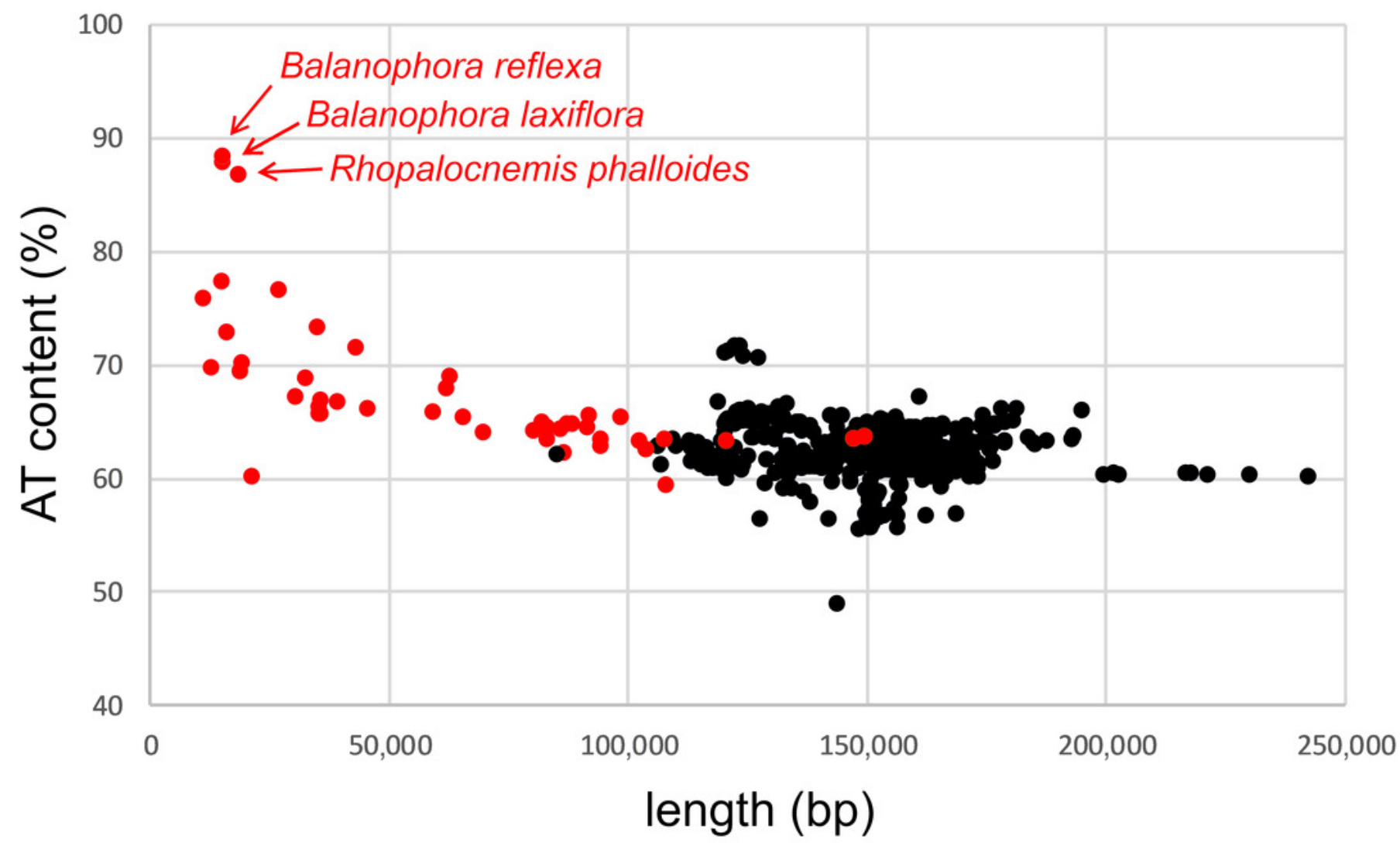


Figure 3

Amino acid frequencies in the plastid proteins of Rhopalocnemis phalloides and Balanophora reflexa are affected by the high AT content.

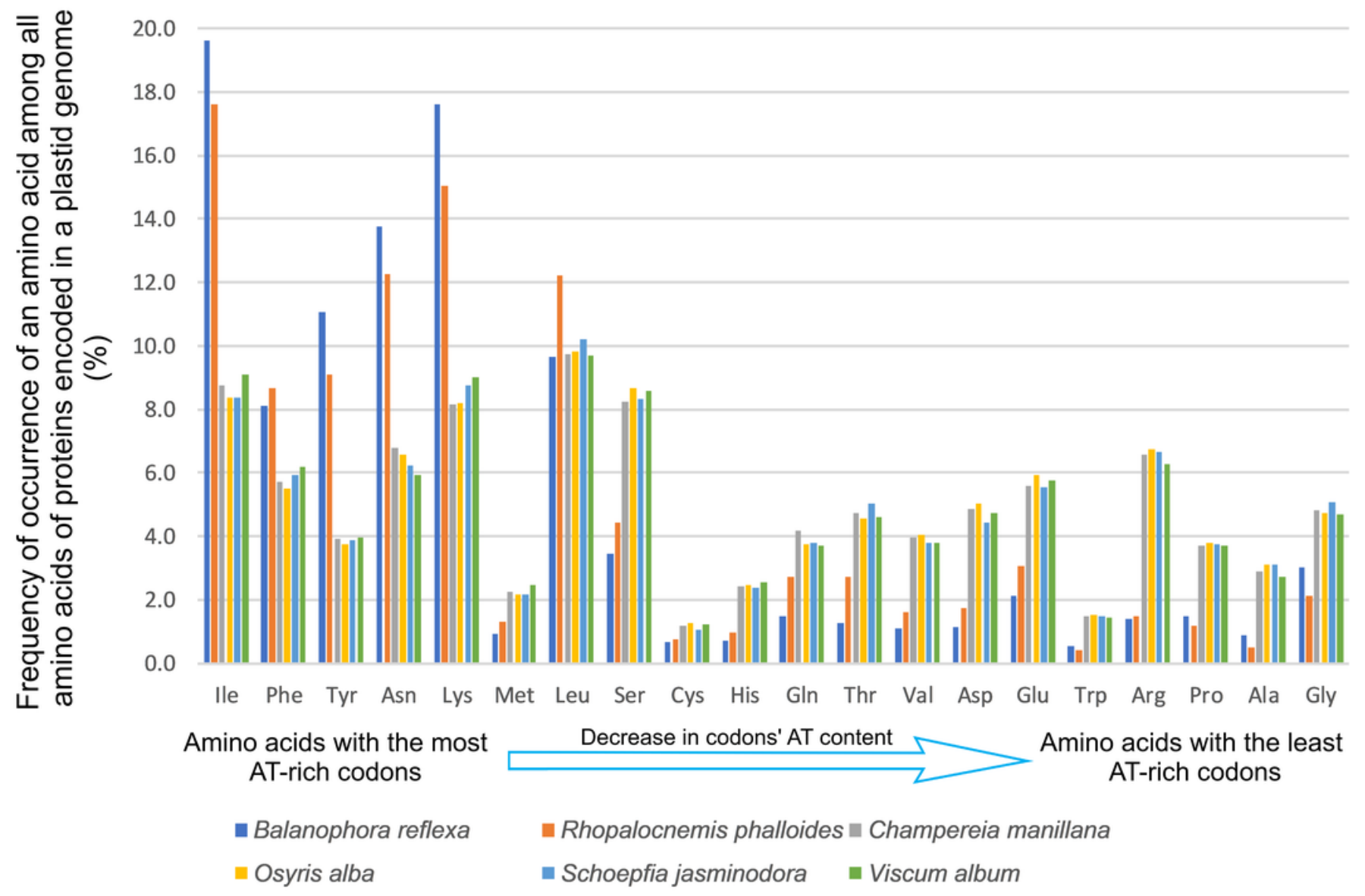


Figure 4 (on next page)

The radial cladogram of rrn16 from Embryophyta and SAR.

Branches with bootstrap support below 70 are collapsed. The group of SAR with Balanophoraceae is coloured orange, while Embryophyta without Balanophoraceae are coloured green. The placement of Balanophoraceae within SAR is indicated by pink. 


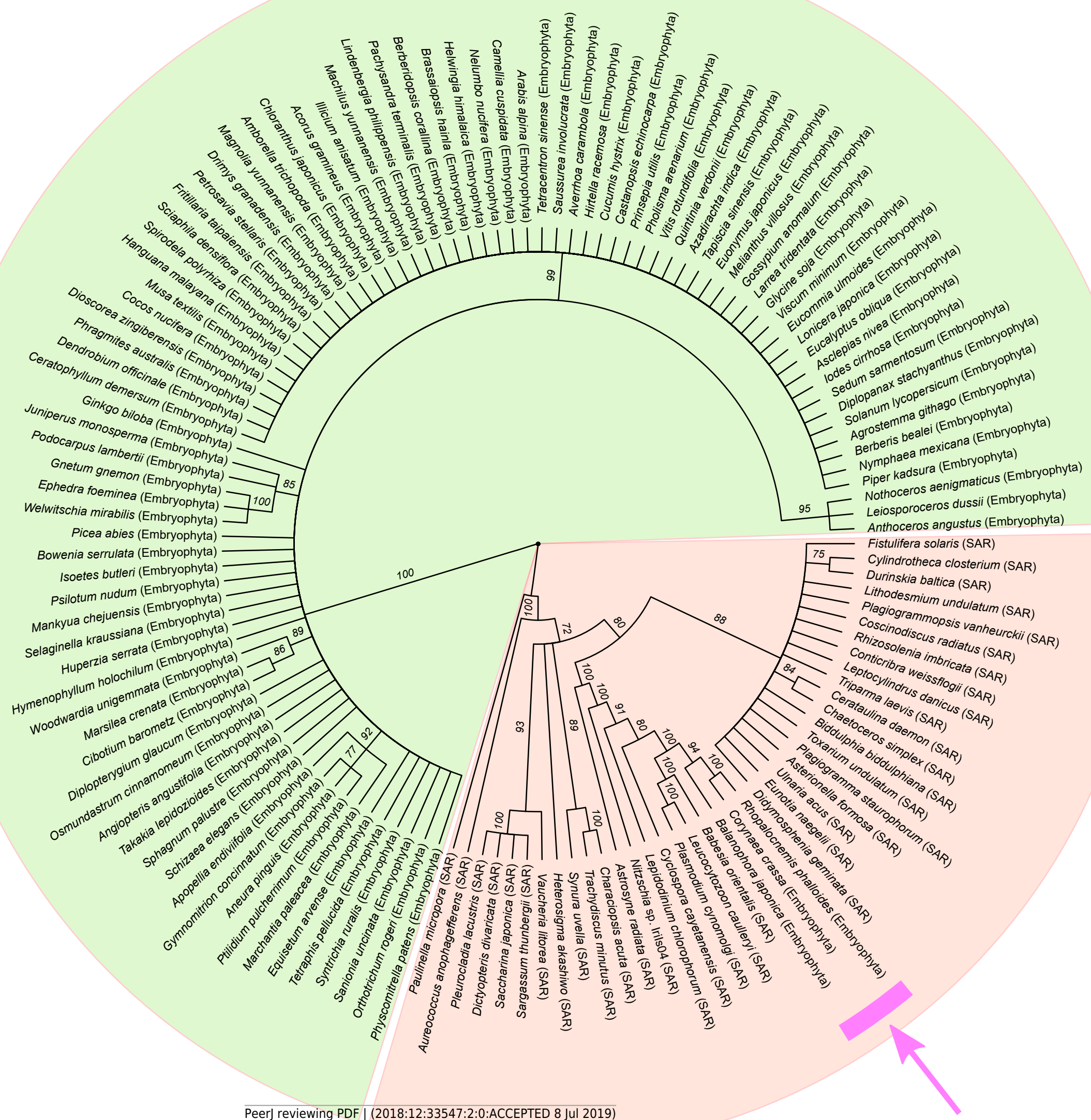


Figure 5

Evolutionary parameters of the phylogeny of Santalales.

Arabidopsis thaliana, used as the outgroup, is not shown. The total length of the alignment, used for the analysis, was 3,363 bp after removal of poorly aligned regions by Gblocks. * $\mathrm{dN} / \mathrm{dS}$ on this branch cannot be calculated owing to a very small dS value. 


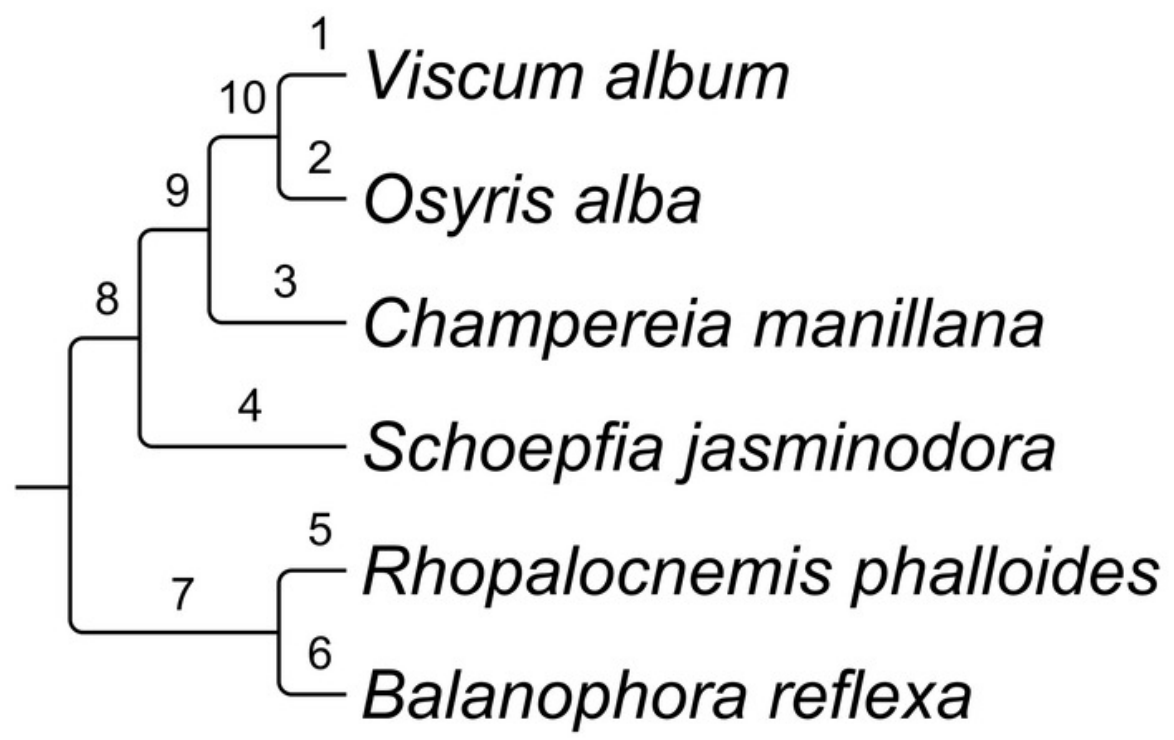

\begin{tabular}{|l|l|l|l|l|}
\hline $\begin{array}{l}\text { Branch } \\
\text { number }\end{array}$ & $\begin{array}{l}\text { Substitutions } \\
\text { per position }\end{array}$ & dN & dS & dN/dS \\
\hline 1 & 0.069 & 0.034 & 0.219 & 0.156 \\
\hline 2 & 0.024 & 0.010 & 0.082 & 0.128 \\
\hline 3 & 0.034 & 0.016 & 0.108 & 0.153 \\
\hline 4 & 0.065 & 0.024 & 0.240 & 0.098 \\
\hline 5 & 0.747 & 0.207 & 3.067 & 0.068 \\
\hline 6 & 0.588 & 0.179 & 2.345 & 0.076 \\
\hline 7 & 0.593 & 0.189 & 2.326 & 0.081 \\
\hline 8 & 0.012 & 0.014 & 0.000 & N/A* \\
\hline 9 & 0.005 & 0.002 & 0.018 & 0.095 \\
\hline 10 & 0.004 & 0.002 & 0.015 & 0.128 \\
\hline
\end{tabular}

